\title{
BCL6B suppresses proliferation and migration of colorectal carcinoma cells through inhibition of the PI3K/AKT signaling pathway
}

\author{
YUE GU, AIFANG LI，HUI SUN，XUERU LI，HE ZHA，JIALI ZHAO, \\ JIAQING XIE, ZONGYUE ZENG and LAN ZHOU
}

Key Laboratory of Diagnostic Medicine Designated by The Chinese Ministry of Education, Chongqing Medical University, Chongqing 400016, P.R. China

Received March 11, 2017; Accepted January 8, 2018

DOI: $10.3892 / \mathrm{ijmm} .2018 .3451$

\begin{abstract}
B-cell CLL/lymphoma 6 member B (BCL6B), a BCL6-homologous gene, has been reported to be a tumor suppressor that is silenced in a variety of human cancers, including colorectal cancer (CRC). Although it was recently demonstrated that reduced expression of BCL6B is associated with tumor stage and lymph node metastasis in CRC, little is known on whether BCL6B contributes to CRC development, or the related underlying mechanism. The aim of the present study was to detect BCL6B expression in CRC cells, and determine the molecular mechanisms underlying the role of BCL6B in CRC development by investigating cell proliferation and migration in vitro. As a result, BCL6B expression was found to be notably repressed in CRC cells compared with normal intestinal epithelial cells by reverse transcription-polymerase chain reaction and western blot analysis. CRC cell proliferation was significantly inhibited by BCL6B upregulation, as indicated by MTT and colony-forming assays. Cell apoptosis was markedly induced, as indicated by flow cytometry, and BCL6B-transfected CRC cells exhibited decreased migration ability. Additionally, BCL6B overexpression diminished the phosphorylation level of AKT in CRC cells. These effects of BCL6B were empowered by treatment with the specific phosphoinositide 3 kinase (PI3K)/AKT inhibitor LY294002. Furthermore, overexpression of BCL6B resulted in upregulation of E-cadherin and downregulation of cyclin D1 and matrix metalloproteinase-9, which were strongly enhanced by LY294002. In conclusion, the findings of the present study
\end{abstract}

Correspondence to: Dr Lan Zhou, Key Laboratory of Diagnostic Medicine Designated by The Chinese Ministry of Education, Chongqing Medical University, 1 Yixueyuan Road, Yuzhong, Chongqing 400016, P.R. China

E-mail: zhoulan@cqmu.edu.cn

Key words: B-cell CLL/lymphoma 6 member B, colorectal cancer, phosphoinositide 3 kinase/AKT signaling pathway, proliferation, migration demonstrated that BCL6B suppressed the proliferation and migration of CRC cells indirectly, via inhibition of PI3K.

\section{Introduction}

Colorectal carcinoma (CRC) is one of the most common malignancies and remains the third leading cause of cancer-related mortality worldwide (1). Although novel molecular-based therapies are widely used for treating CRC, the high recurrence and poor survival rate remain a risk for several CRC patients (2). In recent years, understanding the cancer-specific genes and potential molecular mechanisms underlying the carcinogenesis and progression of $\mathrm{CRC}$ has provided alternative approaches to diagnostic and therapeutic evaluation.

B-cell CLL/lymphoma 6 member B (BCL6B), also referred to as BAZF, ZNF62 and ZBTB28, belongs to the BCL6 gene family and it acts as a sequence-specific transcriptional repressor in the nucleus (3). As regards its biological functions, BCL6B promotes differentiation into stages or lineages in the human erythroleukemia cell line HEL, and plays an important role in spermatogonial stem cell self-renewal $(4,5)$. BCL6B is essential for the secondary responses of memory $\mathrm{CD}^{+} \mathrm{T}$ cells (6). Overexpression of BCL6B induces apoptosis in NIH3T3 cells (7). In recent years, loss of BCL6B expression due to promoter DNA hypermethylation was identified in different types of tumors, including chronic lymphocytic leukemia $(8,9)$, gastric cancer $(10-12)$, hepatocellular carcinoma $(13,14)$ and CRC $(15)$. Methylation of BCL6B has been implicated in tumor growth, angiogenesis, metastasis and invasion (10,13-15). Thus, BCL6B is considered to be a tumor suppressor, and its downregulation may contribute to the development of cancer. However, the precise role and potential molecular mechanism underlying the involvement of BCL6B in CRC progression remain elusive.

The AKT serine/threonine kinase (also referred to as protein kinase $\mathrm{B}$ ), which comprises three highly homologous members, namelyAKT1 $(\mathrm{PKB} \alpha)$, AKT2 $(\mathrm{PKB} \beta)$ and AKT3 $(\mathrm{PKB} \gamma)$, has emerged as a critical signaling molecule within eukaryotic cells (16). AKT is activated in cells exposed to diverse stimuli, such as hormones, growth factors and 
extracellular matrix components. The activation mechanism occurs downstream of phosphoinositide 3-kinase (PI3K), which generates phosphatidylinositol-3,4,5-trisphosphate, a lipid second messenger essential for the translocation of AKT to the plasma membrane, where it is phosphorylated (17). The PI3K/AKT pathway regulates the function of a number of cellular proteins involved in metabolism, proliferation, apoptosis and metastasis (18-20). Previous evidence revealed that the PI3K/AKT pathway is frequently constitutively active in several types of human cancer, including CRC $(21,22)$. However, whether the PI3K/AKT signaling pathway is involved in the BCL6B-induced effects on CRC remains to be elucidated.

Based on the abovementioned studies, the aim of the present study was to investigate the expression, biological role and underlying molecular mechanisms of BCL6B in CRC in order to provide alternative approaches to the treatment of CRC.

\section{Materials and methods}

Cell culture. The human CRC cell lines SW480 and LoVo and the human normal intestinal epithelial cell line FHC were purchased from the American Type Culture Collection (Manassas, VA, USA). The cells were maintained in Dulbecco's modified Eagle's medium (DMEM; Hyclone, Logan, UT, USA) supplemented with $10 \%$ fetal bovine serum (FBS; Gibco; Thermo Fisher Scientific, Carlsbad, CA, USA) and $100 \mathrm{U} / \mathrm{ml}$ streptomycin/penicillin at $37^{\circ} \mathrm{C}$ in a humidified atmosphere containing $5 \% \mathrm{CO}_{2}$.

Reagents. Recombinant plasmid pcDNA3.1-BCL6B and pcDNA3.1 were kindly provided by Dr Xiang (Epigenetics Laboratory, the First Affiliated Hospital of Chongqing Medical University). TRIzol reagent was purchased from Invitrogen; Thermo Fisher Scientific (Carlsbad, CA, USA). Reverse transcription-polymerase chain reaction (RT-PCR) reagents were purchased from Takara (Otsu, Japan). Western blot detection reagents were purchased from the Beyotime Institute of Biotechnology (Nanjing, China). The PI3K/AKT inhibitor LY294002 was purchased from Sigma-Aldrich; Merck KGaA (Saint Louis, MO, USA). The antibodies used were as follows: Rabbit anti-BCL6B polyclonal antibody (cat no. AP20369c, Abgent, Wuxi, China), mouse anti- $\beta$-actin monoclonal antibody (cat no. 47778, Santa Cruz Biotechnology, Dallas, TX, USA), rabbit anti-cyclin D1 polyclonal antibody (cat no. 753, Santa Cruz Biotechnology), mouse anti-matrix metalloproteinase (MMP)-9 monoclonal antibody (cat no. 13520, Santa Cruz Biotechnology), rabbit anti-E-cadherin polyclonal antibody (cat no. YT1454, Immuno Way, TX, USA), rabbit anti-t-AKT monoclonal antibody (cat no. 4691, Cell Signaling Technology, Inc., Danvers, MA, USA), rabbit anti-p-AKT (Ser473) monoclonal antibody (cat no. 81283, Abcam, Boston, MA, USA), goat anti-mouse IgG (cat no. 2305, Zhongshan Golden Bridge, Beijing, China), and goat anti-rabbit IgG (cat no. 2301, Zhongshan Golden Bridge).

Plasmid transfection. The CRC cells were seeded in 6-well plates at a density of $2 \times 10^{5}$ cells per well and transfected with pcDNA3.1-BCL6B (4 $\mu \mathrm{g} /$ well) or the control vector pcDNA3.1
(4 $\mu \mathrm{g} /$ well) using Lipofectamine 2000 (Invitrogen; Thermo Fisher Scientific). After $8 \mathrm{~h}$, the medium was replaced with DMEM containing 10\% FBS followed by continued cell culturing for subsequent experiments.

Cell viability assay. Cell viability was analyzed with the MTT assay. The CRC cells were seeded at a density of $3 \times 10^{3}$ cells per well in 96 -well plates and incubated at $37^{\circ} \mathrm{C}$. At 24, 48, 72 and $96 \mathrm{~h}$ after transfection, and with or without chemical inhibitor in DMEM containing 4\% FBS, $10 \mu \mathrm{l}$ of the 3-(4,5-dimethylthiazol-2-yl)-2,5-diphenyltetrazoliumbromide reagent (MTT; Promega, Madison, WI, USA) was added to each well and incubated for another $4 \mathrm{~h}$ at $37^{\circ} \mathrm{C}$. The culture medium was removed and the formazan was dissolved in DMSO (150 $\mu \mathrm{l} /$ well) for $10 \mathrm{~min}$ at room temperature. The color reaction was measured at $492 \mathrm{~nm}$ with enzyme immunoassay analyzer (Bio-Rad, Hercules, CA, USA). Each condition was carried out in quintuplicate and repeated in at least three batches of independent experiments.

Colony-formation assay. Briefly, at $24 \mathrm{~h}$ post transfection, CRC cells were collected and seeded in 6-well plates (500 cells/well) in $2 \mathrm{ml}$ complete growth medium for $\sim 14$ days, colonies ( $\geq 50$ cells/colony) were fixed with $4 \%$ paraformaldehyde for $15 \mathrm{~min}$ and stained with $0.1 \%$ crystal violet (Beyotime Institute of Biotechnology) for $20 \mathrm{~min}$ and then counted. The experiment was repeated thrice.

Cell apoptosis analysis. Cell apoptosis analysis was assessed by flow cytometry(FCM). CRC cells were collected after transfection for $12 \mathrm{~h}$ and seeded in 6-well plates at a density of $2 \times 10^{5}$ cells/well and starved $12 \mathrm{~h}$ for synchronization, then re-stimulated with $10 \%$ FBS for $24 \mathrm{~h}$. Log-phase cells from each group were harvested by centrifugation. After being washed twice with ice-cold PBS, samples were added into apoptosis analysis solution and then analyzed by a FACS Vantage SE flow cytometer (Becton-Dickinson, San Jose, CA, USA). The experiment was performed three times.

Wound closure assay. Cell migration ability was analyzed with the wound closure assay. Log-phase cells were collected, seeded in 6-well plates and treated with pcDNA3.1-BCL6B or pcDNA3.1. After treating for the indicated time, a wound was created at the center of the culture using a pipette tip, and the cells were washed with serum-free medium, cultured with $1 \%$ FBS. Images were captured at $0,24,48$ and $72 \mathrm{~h}$ under a microscope after the incision was made. The incision width of the different site was measured, and the average wound closure rate was calculated as: $(0 \mathrm{~h}$ incision width- $72 \mathrm{~h}$ incision width $) /(0 \mathrm{~h}$ incision width) $\mathrm{x} 100 \%$. Each experiment was repeated three times.

Transwell assay. Migration: The CRC cells with different treatment were suspended at a density of $5 \times 10^{4}$ in $300 \mu 1$ DMEM containing 10\% FBS and seeded onto the upper chamber of $8.0-\mu \mathrm{m}$ pore size Transwell chambers (Millipore Corporation, Billerica, MA, USA). The lower chamber was filled with $700 \mu \mathrm{l}$ of complete medium containing $20 \% \mathrm{FBS}$ as a chemoattractant. After incubation at $37^{\circ} \mathrm{C}$ for $24 \mathrm{~h}$, the cells that remained in the top chamber were removed with 
cotton swabs. Invasion: The upper chamber was coated with Matrigel (BD Biosciences). SW480 and LoVo cells were added to the upper chamber at a density of $8 \times 10^{4} / 300 \mu 1$ per chamber and incubated for $36 \mathrm{~h}$, followed by removal of the cells that remained in the top chamber with cotton swabs. Cells that penetrated to the lower membrane surface were washed with PBS, fixed in $4 \%$ paraformaldehyde for $20 \mathrm{~min}$ and stained with $0.2 \%$ crystal violet solution, then counted under an inverted microscope (magnification, x100) and photographed. Independent Transwell assay was repeated three times in triplicate.

RNA extraction, RT-PCR and RT- $q P C R$ analysis. Total RNA was extracted from CRC cells in different groups using TRIzol (Invitrogen; Thermo Fisher Scientific), according to the manufacturer's instructions. The RNA concentration and purity were quantified using a NanoDrop 1000 spectrophotometer (Thermo Fisher Scientific). First-strand DNA was synthesized using the Reverse Transcriptase M-MLV (RNase H) kit with random hexamer primers. The primers (Table I) used for RT-PCR and RT-qPCR were designed using the Primer3 Input 0.4.0 (http://frodo.wi.mit.edu/). GAPDH was used as an endogenous control. Touchdown PCR analysis determining the gene expression level was performed under the following conditions: $95^{\circ} \mathrm{C}$ x 5 min for one cycle, 9 cycles at $94^{\circ} \mathrm{C}$ x $30 \mathrm{sec}, 62^{\circ} \mathrm{C}$ x $30 \mathrm{sec}$ (with a decrease of 1 degree/cycle), $55^{\circ} \mathrm{C} \times 30 \mathrm{sec}$ and 25 cycles at $94^{\circ} \mathrm{C} \times 30 \mathrm{sec}, 55^{\circ} \mathrm{C} \times 30 \mathrm{sec}$, and $72^{\circ} \mathrm{C}$ x $30 \mathrm{sec}, 72^{\circ} \mathrm{C}$ x $10 \mathrm{~min}$ for 1 cycle. The PCR products were separated by $2 \%$ agarose gels. The results were recorded by the Gel imaging system (Gel Doc 1000, Bio-Rad) and Quantity One Version 4.5.0 (Bio-Rad). RT-qPCR was run in the Rotor-Gene 6000 Real-Time PCR machine (Corbett Research, Sydney, Australia) using SYBR Premix Ex Taq (Thermo Fisher Scientific) with the following protocol: $95^{\circ} \mathrm{C}$ for $10 \mathrm{~min}$, followed by 40 cycles of denaturation at $95^{\circ} \mathrm{C}$ for $5 \mathrm{sec}$ and an annealing/elongation step at $60^{\circ} \mathrm{C}$ for $20 \mathrm{sec}$. Gene expression results were normalized to GAPDH and analyzed according to the $2^{-\Delta \Delta \mathrm{q}}$ method. Three separate experiments were performed for each group.

Western blot assay. Cells with different treatments were collected and washed with ice-cold PBS, then lysed in ice-cold RIPA lysis buffer (Beyotime Institute of Biotechnology, Shanghai, China) containing protease inhibitor and phosphatase inhibitor (Roche, Mannheim, Germany). Protein extracts were quantitated by the bicinchoninic acid assay, denatured by boiling, separated by $10 \%$ sodium dodecyl sulfate polyacrylamide gel electrophoresis and blotted onto the PVDF membranes. After blocking with $5 \%$ bovine serum albumin (Solarbio, Beijing, China) at room temperature for $2 \mathrm{~h}$ in Tris-buffered saline and Tween 20 (TBST), the membranes were probed with the primary antibodies $(1: 1,000)$ and incubated at $4^{\circ} \mathrm{C}$ overnight. Subsequently, the membranes were washed with TBST and incubated with horseradish peroxidase-conjugated secondary antibodies $(1: 5,000)$ for $1 \mathrm{~h}$. After washing with TBST, the proteins were detected using enhanced chemiluminescence (Millipore Corporation), followed by exposure on the Gel Doc 1000 Electrophoresis Documentation (Bio-Rad). Three separate experiments were performed for each group.
Table I. Primer sequences in RT-PCR.

\begin{tabular}{ll}
\hline Gene & \multicolumn{1}{c}{ Primer sequences } \\
\hline GAPDH & F: 5'-CAGCGACACCCACTCCTC-3' \\
& R: 5'-TGAGGTCCACCACCCTGT-3' \\
BCL6B & F:5'-AAGCCGTATAAGTGTGAGACG-3' \\
& R: 5'-AGAATGTGGTAGTGCAC-3' \\
Cyclin D1 & F: 5'-CTGGCCATGAACTACCTGGA-3' \\
& R: 5'-GTCACACTTGATCACTCTGG-3' \\
E-cadherin & F:5'-AAGGTGACAGAGCCTCTGGAT-3' \\
& R: 5'-CGTCTGTGGCTGTGACCT-3' \\
MMP-9 & F:5'-CCTGGAGCCTGAGAACCAATC-3' \\
& R:5'-CCACCCGAGTGTAACCATGGC-3'
\end{tabular}

RT-PCR, reverse transcription-polymerase chain reaction; BCL6B, B-cell CLL/lymphoma 6 member B; MMP, matrix metalloproteinase.

Statistical analysis. All quantitative data were calculated and are presented as means \pm standard deviation. The difference between two different groups was determined by Student's $\mathrm{t}$-test and the differences between different groups were analyzed using one-way analysis of variance followed by the Student-Newman-Keuls test. All the statistical analyses were performed using GraphPad Prism 5 (GraphPad Software, La Jolla, CA, USA). Statistical significance was set at $\mathrm{P}<0.05$.

\section{Results}

BCL6B is downregulated in human CRC cells. The mRNA expression of BCL6B was first compared between the human normal intestinal epithelial FHC cells and the two CRC cell lines SW480 and LoVo (Fig. 1A and B). BCL6B was readily expressed in FHC cells, but was notably repressed in SW480 and LoVo cells. Western blot analysis confirmed the loss of BCL6B protein expression in SW480 and LoVo cells (Fig. 1C), suggesting that BCL6B may play an important role in CRC.

BCL6B overexpression suppresses proliferation of CRC cells. To investigate the biological role of BCL6B in CRC, SW480 and LoVo cells were transfected with pcDNA3.1-BCL6B for BCL6B overexpression (Fig. 2A). Cell viability and proliferation were analyzed with the MTT assay and the colony formation assay. Compared with the control group, BCL6B overexpression strongly decreased the viability of SW480 and LoVo cells at 72 and $96 \mathrm{~h}$ (Fig. 2B). In addition, the colony numbers of SW480 or LoVo cells in the BCL6B group were significantly lower compared with those in the control group (Fig. 2C).

BCL6B overexpression induces apoptosis of CRC cells. To determine whether the observed growth inhibition induced by BCL6B was associated with cell apoptosis, FCM was applied. As a result, the rate of apoptotic cells was $21.92 \pm 3.32$ vs. $12.51 \pm 2.61 \%(\mathrm{P}<0.05)$, and $21.37 \pm 3.46$ vs $11.21 \pm 1.28 \%(\mathrm{P}<0.05)$ in $\mathrm{SW} 480$ and LoVo cells with and without overexpression of BCL6B, respectively (Fig. 3). These data suggested that BCL6B promoted apoptosis in CRC cells. 

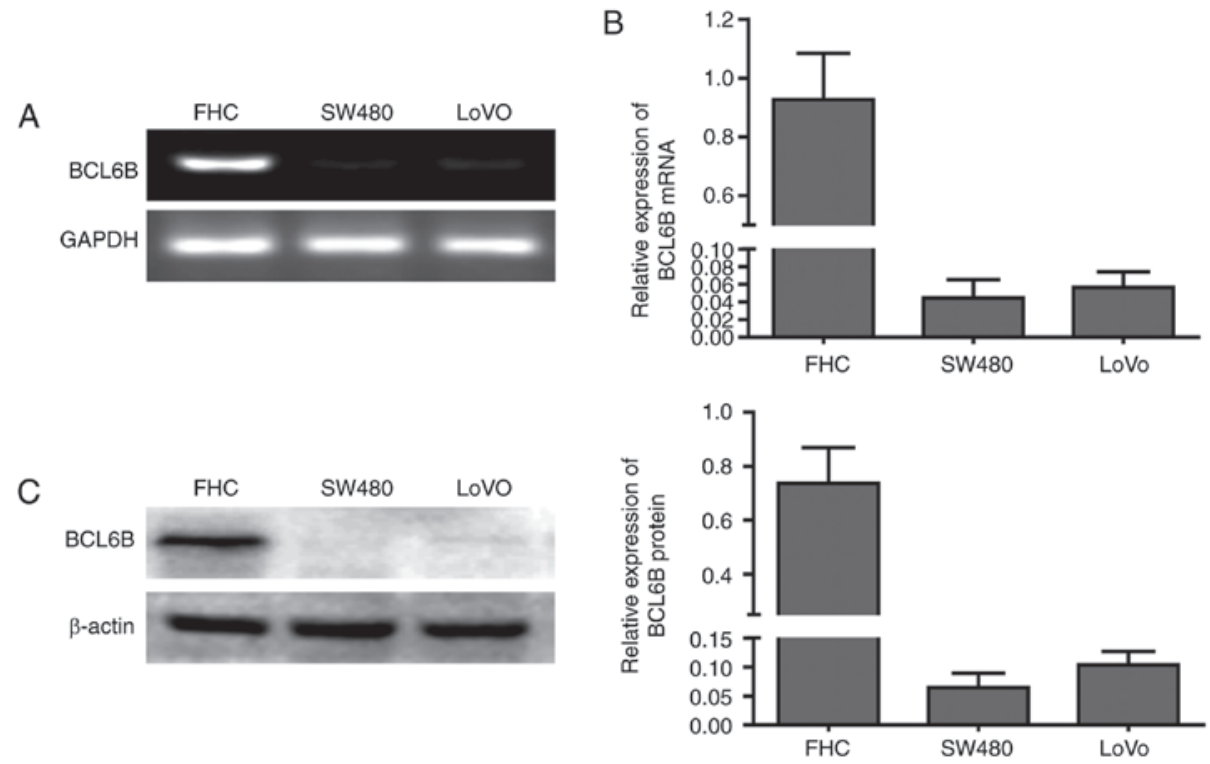

Figure 1. BCL6B expression is notably decreased in CRC cells. (A) BCL6B mRNA in FHC cells and CRC cells was detected by RT-PCR. (B) BCL6B mRNA was detected by RT-qPCR. Data are reported as mean values \pm SD of three individual measurements. (C) BCL6B protein was detected by western blot analysis. Data are reported as mean values \pm SD of three individual measurements. BCL6B, B-cell CLL/lymphoma 6 member B; CRC, colorectal cancer; RT-qPCR, reverse transcription-quantitative polymerase chain reaction; SD, standard deviation.
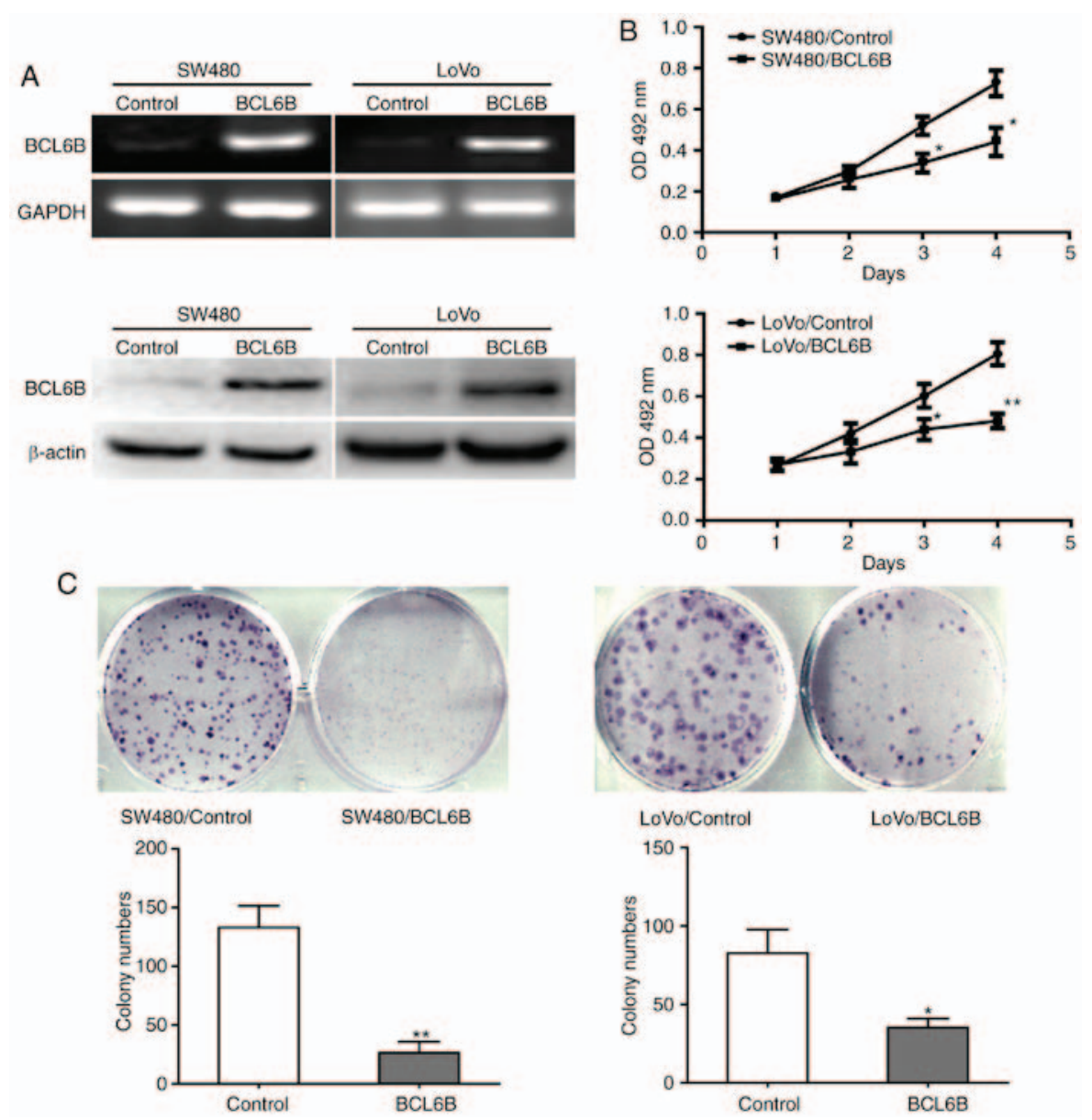

Figure 2. BCL6B inhibits proliferation of CRC cells. (A) Increased mRNA and protein level of BCL6B in SW480 and LoVo cells following transfection with pcDNA3.1-BCL6B for $48 \mathrm{~h}$ were detected by RT-PCR and western blot analysis. (B) Growth curves represent MTT assay results of 4 days for SW480 and LoVo cells transfected with pcDNA3.1 or pcDNA3.1-BCL6B. The results show the mean absorbance \pm SD of three independent experiments $\left(\right.$ "P $<0.05,{ }^{* * *} \mathrm{P}<0.01 \mathrm{vs}$. control group). (C) The effect of BCL6B on the proliferation of SW480 and LoVo cells was detected with the colony formation assay. The number of colonies decreased significantly in the BCL6B group in SW480 and LoVo cells. Representative images of the colony-forming unit are presented in the upper panel, and average colony numbers for each group were calculated and are presented in the lower panel. Each assay condition was carried out in triplicate and repeated in at least three batches of independent experiments ( $\mathrm{P}<0.05,{ }^{* *} \mathrm{P}<0.01$ vs. control group). BCL6B, B-cell CLL/lymphoma 6 member B; CRC, colorectal cancer; RT-PCR, reverse transcription-polymerase chain reaction; SD, standard deviation. 

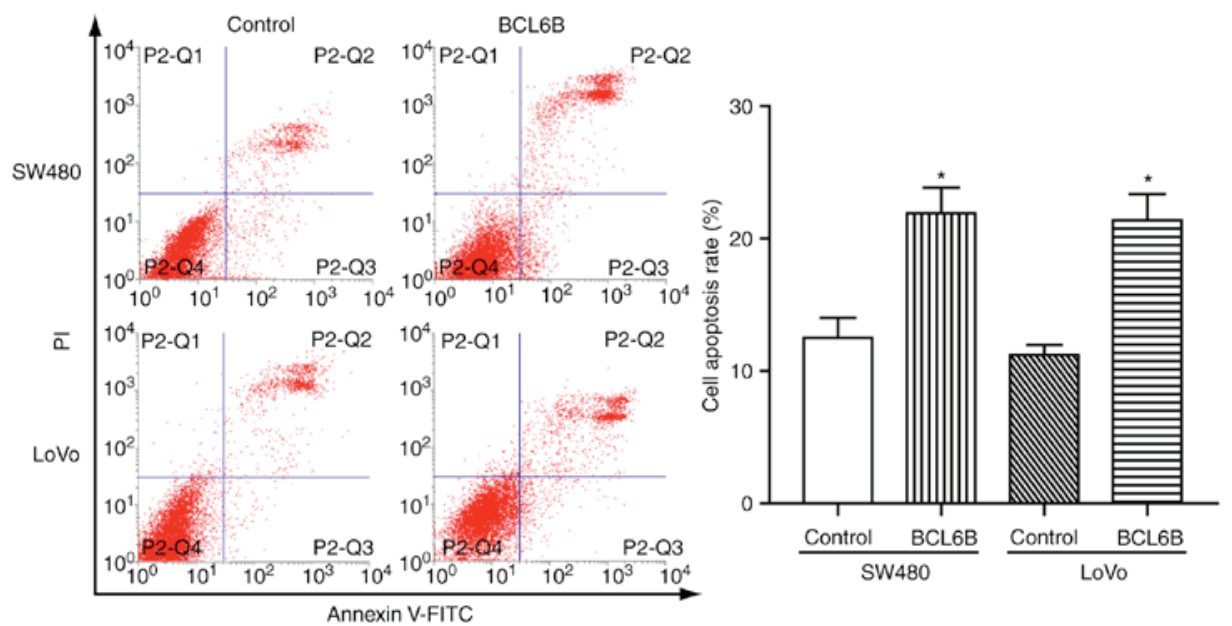

Figure 3. BCL6B induces apoptosis in CRC cell lines. SW480 and LoVo cells were transfected with pcDNA3.1-BCL6B for 48 h and cell apoptosis was analyzed by flow cytometry. The percentage of cells for each group were quantified and presented in the right panel. Values are the means $\pm \mathrm{SD}(\mathrm{n}=3)($ ( $\mathrm{P}<0.05$ vs. control group). BCL6B, B-cell CLL/lymphoma 6 member B; CRC, colorectal cancer; SD, standard deviation.
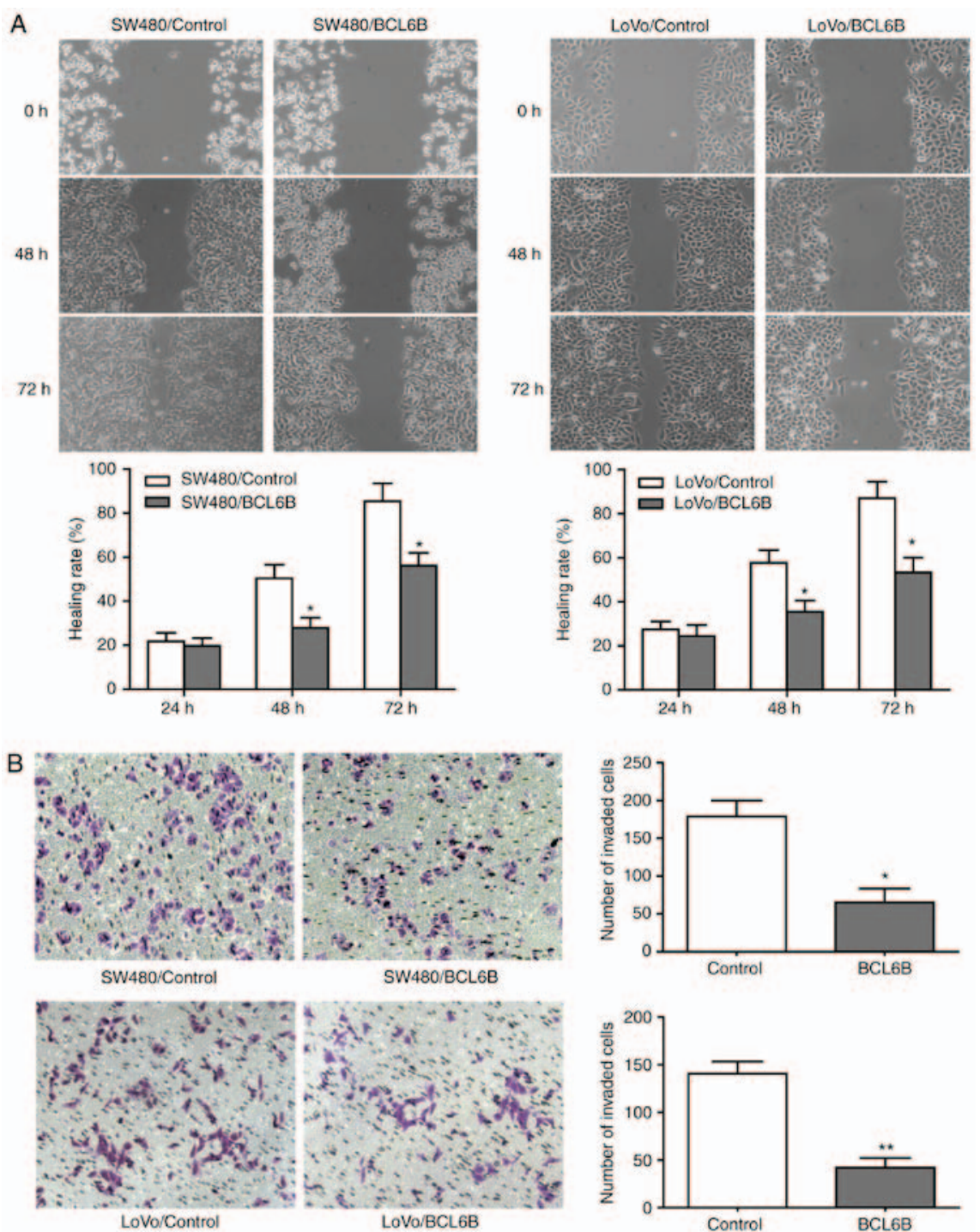

Figure 4. BCL6B suppresses migration and invasion of SW480 and LoVo cells. (A) Wound closure assay of CRC cells transfected with pcDNA3.1 or pcDNA3.1-BCL6B. Representative images are shown in the upper panel (magnification, x100). The wound closure rates for each group are quantified in the lower panel. The wound closure rate was obtained as mentioned in Materials and methods. The experiment was repeated three times ("P $<0.05$ vs. control group). (B) Transwell invasion assay of CRC cells transfected with pcDNA3.1or pcDNA3.1-BCL6B. Representative images are shown in the left panel (magnification, x100). The average number of invading cells for each group were calculated and presented in the right panel. The experiment was repeated three times in triplicate ( $\mathrm{P}<0.05$ and ${ }^{* *} \mathrm{P}<0.01$ vs. control group). BCL6B, B-cell CLL/lymphoma 6 member B; CRC, colorectal cancer. 

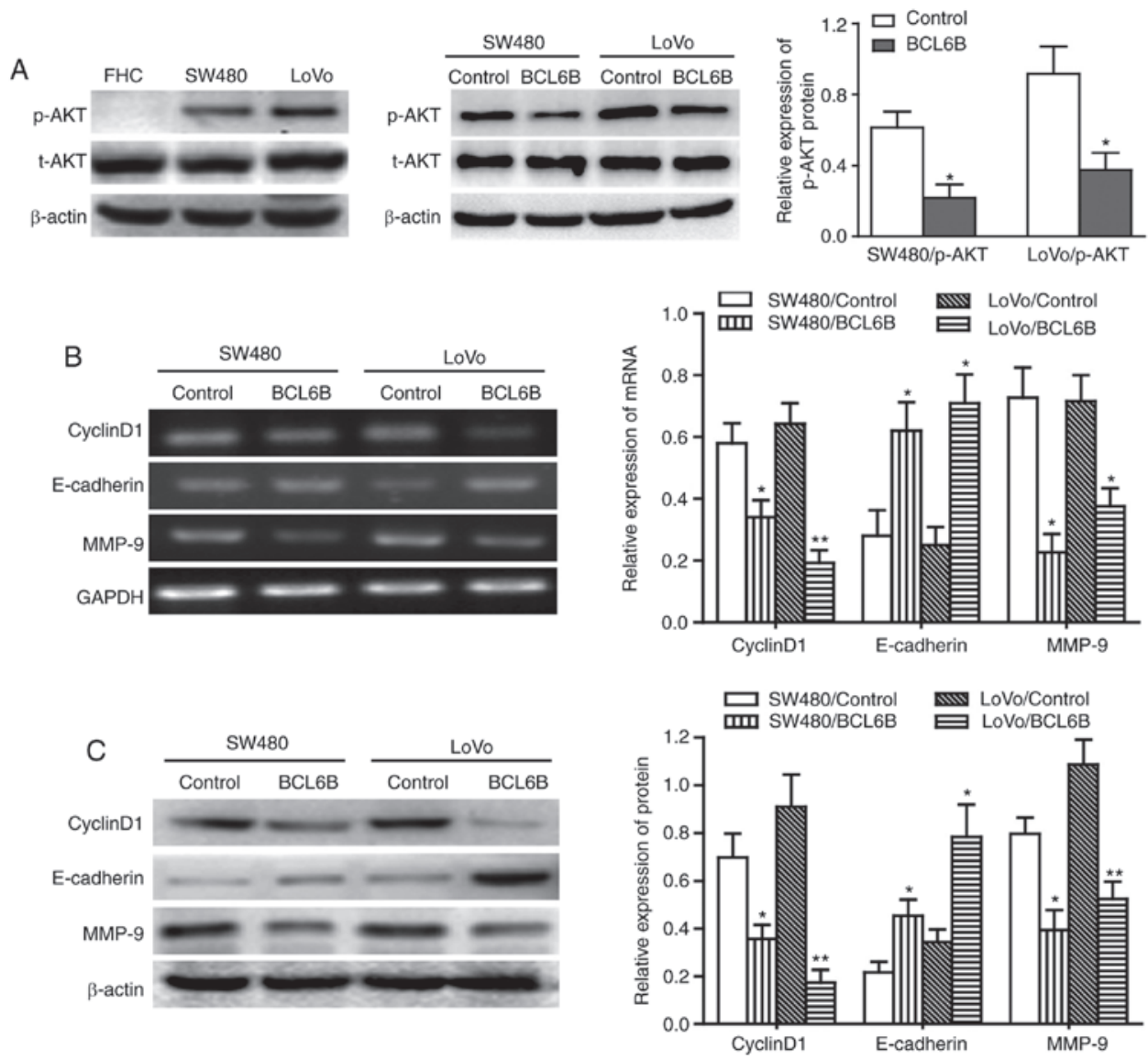

Figure 5. BCL6B inhibits the PI3K/AKT pathway, and increases E-cadherin expression and decreases cyclin D1 and MMP-9 expression in CRC cells. (A) The basal phosphorylation level of AKT in the CRC cell lines SW480 and LoVo was notably elevated compared with that in the normal intestinal epithelial cell line FHC, and the phosphorylation level of AKT was significantly decreased in BCL6B-overexpressing SW480 and LoVo cells, which were detected by western blot analysis. Data are shown as mean values $\pm \mathrm{SD}$ of three individual measurements (" $\mathrm{P}<0.05$ vs. control group). (B) RT-PCR and (C) western blot assays were performed to measure the expression of E-cadherin, cyclin D1 and MMP-9 in CRC cells. Densitometric quantification data are presented on the right panel Data are shown as mean values \pm SD of three individual measurements ( $\mathrm{P}<0.05$ and ${ }^{* *} \mathrm{P}<0.01$ vs. control group). BCL6B, B-cell CLL/lymphoma 6 member B; CRC, colorectal cancer; MMP, matrix metalloproteinase; RT-PCR, reverse transcription-polymerase chain reaction; SD, standard deviation.

BCL6B overexpression inhibits migration and invasion of CRC cells. As shown in Fig. 4A, the wound closure rate of SW480 and LoVo cells in the BCL6B overexpression group was decreased by $38.89 \%(\mathrm{P}<0.05)$ and $31.17 \%(\mathrm{P}<0.05)$, respectively, at $48 \mathrm{~h}$. In addition, $36 \mathrm{~h}$ after transfection, the numbers of invading SW480 and LoVo cells in the BCL6B overexpression group were decreased by $63.69 \%(\mathrm{P}<0.05)$ and $70.71 \%(\mathrm{P}<0.01)$, respectively (Fig. 4B).

BCL6B inhibits the PI3K/AKT signaling pathway in CRC cells. We next sought to determine the signaling mechanism involved in BCL6B-induced decrease in the proliferation and migration of CRC cells by focusing on the PI3K/AKT signaling pathway, which plays a critical role in cell proliferation, migration and cancer progression. Our data demonstrated that the basal phosphorylation level of AKT in the CRC cell lines SW480 and LoVo was significantly higher compared with that in the normal intestinal epithelial cell line FHC, and that the phosphorylation level of AKT was markedly decreased in these two CRC cell lines with BCL6B overexpression $(\mathrm{P}<0.05)$, whereas there was no significant change in the total AKT level (Fig. 5A). cyclin D1 and MMP-9 expression in CRC cells. Previous studies demonstrated that the expression of cyclin D1, E-cadherin and MMP-9 was regulated by the PI3K/AKT signaling pathway, and cyclin D1, E-cadherin and MMP-9 have been found to be involved in the proliferation and migration of various tumor cells (23-26). We next examined the effect of BCL6B on the expression of cyclin D1, E-cadherin and MMP-9. The results revealed that BCL6B overexpression upregulated E-cadherin and downregulated cyclin D1 and MMP-9 in SW480 and LoVo cells, at the mRNA as well as the protein levels (Fig. 5B and C).

BCL6B suppresses cell proliferation and migration indirectly via inhibition of PI3K. To further investigate how BCL6B acts on the PI3K/AKT signaling pathway, BCL6B-overexpressing LoVo cells were treated with $10 \mu \mathrm{M}$ of the PI3K/AKT inhibitor LY294002, and cell viability and migration were measured at the indicated time points by the MTT assay and Transwell chamber assay, respectively. As a result, our data revealed that the decreased proliferation and migration abilities and the repressed AKT activity caused by either BCL6B overexpression or LY294002 in LoVo cells were both notably enhanced by combined treatment with LY294002 and BCL6B (Fig. 6A-C). Furthermore, the upregulated expression of E-cadherin and downregulated expression of cyclin D1 and MMP-9 induced by BCL6B in LoVo cells were enhanced by 

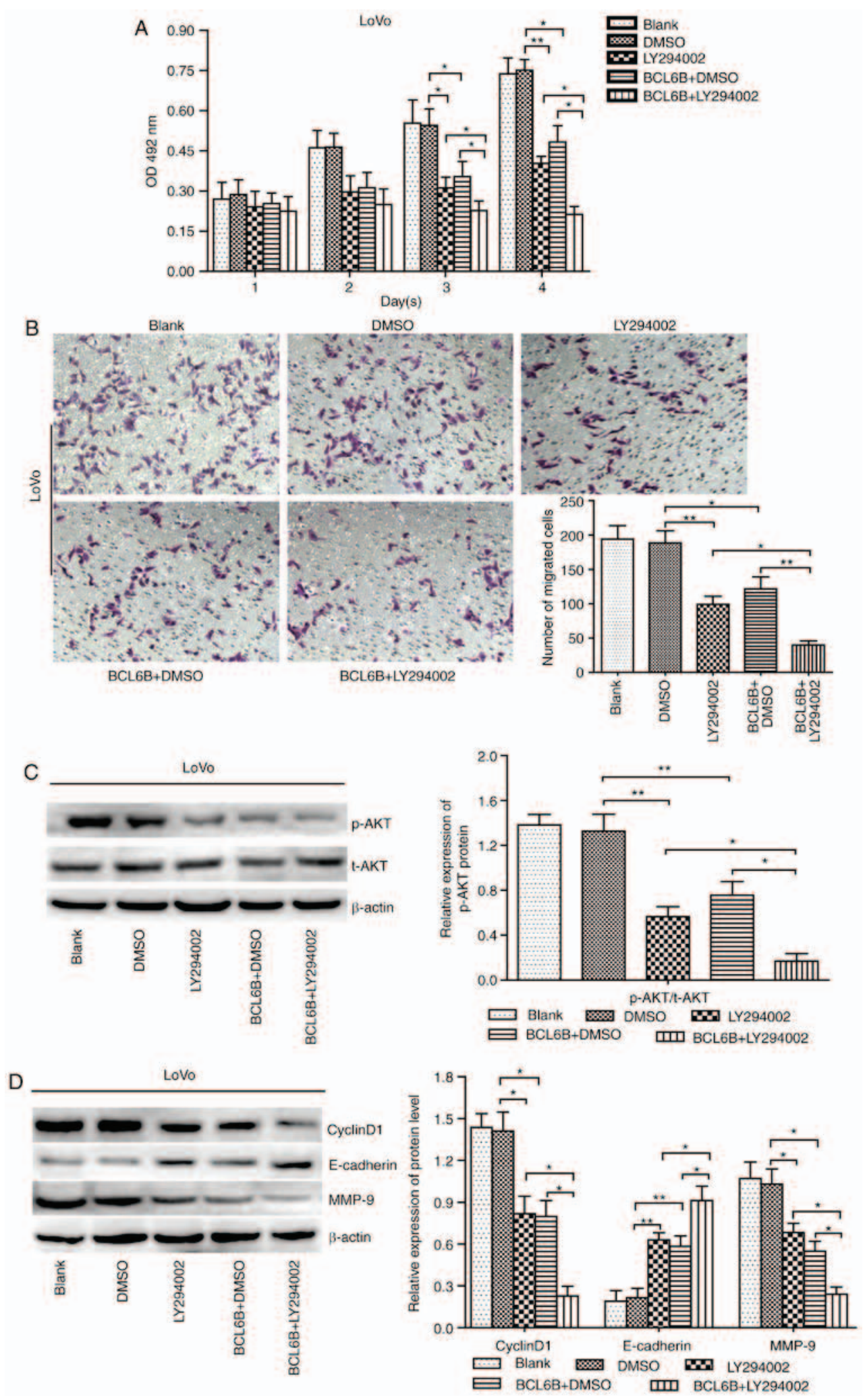

Figure 6. LY294002 enhances BCL6B-induced inhibition of the proliferation and migration of LoVo cells. (A) Effect of LY294002 on BCL6B-induced inhibition of viability of LoVo cells. Cell viability was measured with the MTT assay. The inhibitory role of BCL6B in cell proliferation was enhanced by LY294002. The results show the mean absorbance \pm SD of three independent experiments ("P<0.05 vs. LY294002 group, ${ }^{* *} \mathrm{P}<0.01$ vs. BCL6B + DMSO group). (B) Effect of LY294002 on BCL6B-induced inhibition of migration of LoVo cells, which was measured by the Transwell assay (magnification, x100). The inhibitory role of BCL6B in cell migration was enhanced by LY294002 ("P<0.05 vs. LY294002 group, ${ }^{* *} \mathrm{P}<0.01$ vs. BCL6B + DMSO group). (C) Effect of LY294002 on BCL6B-induced suppression of AKT in LoVo cells was detected by western blot analysis. The decreased expression of p-AKT induced by BCL6B was enhanced by LY294002. Densitometric quantification data are on the right panel. Data are shown as mean values \pm SD of three individual measurements ( ${ }^{* *} \mathrm{P}<0.01$ vs. DMSO group, ${ }^{*} \mathrm{P}<0.05$ vs. LY294002 group and BCL6B + DMSO group). (D) Effects of LY294002 on BCL6B-induced changes in the protein levels of cyclin D1, E-cadherin and MMP-9 in LoVo cells were detected by western blot analysis. The expression levels of cyclin D1, E-cadherin and MMP-9 was enhanced by LY294002. Densitometric quantification data are presented on the right panel. Data are shown as mean values \pm SD of three individual measurements ("P<0.05 vs. LY294002 group, "P<0.05 vs. BCL6B+DMSO group). BCL6B, B-cell CLL/lymphoma 6 member B; OD, optical density; SD, standard deviation; DMSO, dimethyl sulfoxide; MMP, matrix metalloproteinase. 
LY294002 (Fig. 6D). These findings suggested that BCL6B suppresses proliferation and migration of CRC cells indirectly, via inhibition of PI3K.

\section{Discussion}

In the present study, the mRNA and protein expression of BCL6B were found to be markedly repressed in the CRC cell lines SW480 and LoVo compared with that in the normal intestinal epithelial cell line FHC. In addition, BCL6B overexpression inhibited cancer cell proliferation, colony formation, migration and invasion, whereas it promoted cell apoptosis. Mechanistically, these findings provide evidence showing that the basal phosphorylation level of AKT in the SW480 and LoVo cells was observably elevated compared with that in FHC cells, and that BCL6B overexpression reduced AKT phosphorylation, cyclin D1 and MMP-9, and increased E-cadherin. More importantly, these effects of BCL6B on CRC cells were enhanced by the PI3K/AKT inhibitor LY294002. These results suggest that BCL6B suppresses the proliferation and migration of CRC cells through inhibition of the PI3K, in an indirect manner.

Over the past decades, molecular genetic studies have revealed that epigenetic alterations, particularly inactivation of tumor suppressor genes or tumor-related genes through promoter hypermethylation, play an important role in the tumorigenesis and development of CRC (27-30). Emerging evidence suggests that BCL6Bwas silenced or downregulated due to promoter DNA hypermethylation in a variety of human cancers (8). Clinical evidences indicated that loss of BCL6B expression was associated with cancer progression, metastasis and survival (10-15). Consistent with previous reports, we found that the endogenous expression of BCL6B in the CRC cell lines SW480 and LoVo was significantly lower compared with that in the human normal intestinal epithelial cell line FHC. Therefore, SW480 and LoVo cells were transfected with pcDNA3.1-BCL6B to investigate the biological role and molecular basis of BCL6B in CRC.

Our data revealed that SW480 and LoVo cells with BCL6B overexpression exhibited significantly decreased viability and colony-forming ability. A possible explanation for the growth inhibition is cell cycle arrest or increase in apoptosis. The results derived from FCM demonstrated that BCL6B induced apoptosis of SW480 and LoVo cells. In addition, we observed that cyclin D1, an important regulator of the cell cycle, was downregulated at both the mRNA and protein levels, further confirming that BCL6B inhibits CRC cell proliferation. Previous studies have reported that BCL6B can suppress cancer cell growth, invasion and metastasis $(10,13,15)$. For example, BCL6B inhibits hepatocellular carcinoma metastasis and angiogenesis through the upregulation of E-cadherin and downregulation of vascular endothelial growth factor (13). Restoration of BCL6B expression suppresses CRC cell growth and migration by activating P53 signaling (15). In agreement with these studies, the results of the wound closure assay and Transwell assay revealed that SW480 and LoVo cells with BCL6B overexpression exhibited decreased migration and invasion abilities. In addition, we observed that the mRNA and protein level of the EMT-related marker E-cadherin was increased and that the expression of the tumor metastasis-related gene MMP-9 was decreased. Upregulation of E-cadherin and downregulation of MMP-9 by BCL6B may enhance intercellular adhesions, thus inhibiting tumor cell migration (31).

The PI3K/AKT signaling pathway plays a pivotal role in cell proliferation, apoptosis, survival, migration and invasion (21). A number of studies indicated that aberrant activation of the PI3K/AKT signaling pathway is associated with human CRC $(22,32)$. AKT kinase activity is induced following activation of PI3K in growth factor receptor-mediated signaling cascades (33). Previous findings demonstrated frequent alterations inPI3K/AKT signaling in human CRC $(33,34)$. The PI3K/AKT pathway regulates cyclin D1, E-cadherin and MMP-9 expression, thus affecting cell proliferation and migration in different tumors $(24,35,36)$. In the present study, the data demonstrated that the basal AKT activity in SW480 and LoVo cells was significantly higher compared with that in the normal intestinal epithelial cell line FHC, and that BCL6B overexpression effectively reduced the phosphorylation level of AKT in SW480 and LoVo cells. Moreover, the decreased proliferation and migration ability and repressed AKT activity caused by either BCL6B overexpression or the PI3K/AKT inhibitor LY294002 in LoVo cells were both notably enhanced by combined treatment with LY294002 and BCL6B. Furthermore, the upregulation of E-cadherin and downregulation of cyclin D1 and MMP-9 by BCL6B in LoVo cells were enhanced by LY294002. These results demonstrated that BCL6B suppresses proliferation and migration of CRC cells indirectly, via inhibition of PI3K, and the mechanism underlying the effects of BCL6B on the PI3K/AKT pathway requires further investigation. In conclusion, the present study demonstrated that BCL6B, which is downregulated in CRC cells, acts as a tumor suppressor in CRC by suppressing cell proliferation, migration and invasion through the inhibition of PI3K/AKT signaling pathway. Therefore, BCL6B overexpression may prove to be a valuable tool for inhibiting cancer growth. However, future studies are required to elucidate the association between BCL6B and the PI3K/AKT pathway in CRC.

\section{Acknowledgements}

The authors would like to thank Professor Xiang (Epigenetics Laboratory, The First Affiliated Hospital of Chongqing Medical University) for her kind provision of the recombinant plasmids pcDNA3.1-BCL6B and pcDNA3.1.

\section{Competing interests}

The authors declare that they have no competing interests.

\section{References}

1. Siegel R, Naishadham D and Jemal A: Cancer statistics, 2010. CA Cancer J Clin 61: 10-29, 2011.

2. Meyerhardt JA, Li L, Sanoff HK, Carpenter W IV and Schrag D: Effectiveness of bevacizumab with first-line combination chemotherapy for Medicare patients with stage IV colorectal cancer. J Clin Oncol 30: 608-615, 2012.

3. Okabe S, Fukuda T, Ishibashi K, Kojima S, Okada S, Hatano M, Ebara M, Saisho H and Tokuhisa T: BAZF, a novel Bcl6 homolog, functions as a transcriptional repressor. Mol Cell Biol 18: 4235-4244, 1998. 
4. Sakashita C, Fukuda T, Okabe S, Kobayashi H, Hirosawa S, Tokuhisa T, Miyasaka N, Miura O and Miki T: Cloning and characterization of the human BAZF gene, a homologue of the BCL6 oncogene. Biochem Biophys Res Commun 291: 567-573, 2002.

5. Oatley JM, Avarbock MR, Telaranta AI, Fearon DT and Brinster RL: Identifying genes important for spermatogonial stem cell self-renewal and survival. Proc Natl Acad Sci USA 103 9524-9529, 2006.

6. Manders PM, Hunter PJ, Telaranta AI, Carr JM, Marshall JL, Carrasco M, Murakami Y, Palmowski MJ, Cerundolo V, Kaech SM, et al: BCL6b mediates the enhanced magnitude of the secondary response of memory CD8+ T lymphocytes. Proc Natl Acad Sci USA 102: 7418-7425, 2005.

7. Zhang H, Okada S, Hatano M, Okabe S and Tokuhisa T: A new functional domain of Bcl6 family that recruits histone deacetylases. Biochim Biophys Acta 1540: 188-200, 2001.

8. Ying J, Srivastava G, Hsieh WS, Gao Z, Murray P, Liao SK, Ambinder R and Tao Q: The stress-responsive gene GADD45G is a functional tumor suppressor, with its response to environmental stresses frequently disrupted epigenetically in multiple tumors. Clin Cancer Res 11: 6442-6449, 2005.

9. Greipp PT, Smoley SA, Viswanatha DS, Frederick LS, Rabe KG, Sharma RG, Slager SL, Van Dyke DL, Shanafelt TD, Tschumper RC and Zent CS: Patients with chronic lymphocytic leukaemia and clonal deletion of both 17p13.1 and 11q22.3 have a very poor prognosis. Br J Haematol 163: 326-333, 2013.

10. Xu L, Li X, Chu ES, Zhao G, Go MY, Tao Q, Jin H, Zeng Z, Sung JJ and $\mathrm{Yu}$ J: Epigenetic inactivation of BCL6B, a nove functional tumour suppressor for gastric cancer, is associated with poor survival. Gut 61: 977-985, 2012.

11. Yang Q, Gao J, Xu L, Zeng Z, Sung JJ and Yu J: Promoter hypermethylation of BCL6B gene is a potential plasma DNA biomarker for gastric cancer. Biomarkers 18: 721-725, 2013.

12. Deng J, Han L, Dong Q, Hou Y, Xie X, Yu J, Fan D and Hao X: The survival decrease in gastric cancer is associated with the methylation of B-cell CLL/lymphoma 6 member B promoter. Open Biol 4: 140067, 2014.

13. Wang J, Dong L, Xu L, Chu ES, Chen Y, Shen J, Li X, Wong CC, Sung JJ and Yu J: B cell CLL/lymphoma 6 member B inhibits hepatocellular carcinoma metastases in vitro and in mice. Cancer Lett 355: 192-200, 2014.

14. Li X, Yu J, Brock MV, Tao Q, Herman JG, Liang P and Guo M: Epigenetic silencing of BCL6B inactivates p53 signaling and causes human hepatocellular carcinoma cell resist to 5-FU. Oncotarget 6: 11547-11560, 2015.

15. Hu S, Cao B, Zhang M, Linghu E, Zhan Q, Brock MV, Herman JG, Mao G and Guo M: Epigenetic silencing BCL6B induced colorectal cancer proliferation and metastasis by inhibiting P53 signaling. Am J Cancer Res 5: 651-662, 2015.

16. Franke TF: PI3K/Akt: Getting it right matters. Oncogene 27: 6473-6488, 2008.

17. Nicholson KM and Anderson NG: The protein kinase B/Akt signalling pathway in human malignancy. Cell Signal 14: 381-395, 2002.

18. Michl P and Downward J: Mechanisms of disease: PI3K/AKT signaling in gastrointestinal cancers.ZGastroenterol43: 1133-1139, 2005.

19. Kawauchi K, Ogasawara T, Yasuyama M, Otsuka K and Yamada O: The PI3K/Akt pathway as a target in the treatment of hematologic malignancies. Anticancer Agents Med Chem 9: 550-559, 2009.

20. Manfredi GI, Dicitore A, Gaudenzi G, Caraglia M, Persani L and Vitale G: PI3K/Akt/mTOR signaling in medullary thyroid cancer: A promising molecular target for cancer therapy. Endocrine 48: 363-370, 2015.
21. Vivanco I and Sawyers CL: The phosphatidylinositol 3-Kinase AKT pathway in human cancer. Nat Rev Cancer 2: 489-501, 2002.

22. Rychahou PG, Jackson LN, Silva SR, Rajaraman S and Evers BM: Targeted molecular therapy of the PI3K pathway: Therapeutic significance of PI3K subunit targeting in colorectal carcinoma. Ann Surg 243: 833-844, 2006.

23. Gao N, Zhang Z, Jiang BH and Shi X: Role of $\mathrm{PI} 3 \mathrm{~K} / \mathrm{AKT} / \mathrm{mTOR}$ signaling in the cell cycle progression of human prostate cancer. Biochem Biophys Res Commun 310: 1124-1132, 2003

24. Song G, Ouyang G and Bao S: The activation of AKT/PKB signaling pathway and cell survival. J Cell Mol Med 9: 59, 2005.

25. Zang M, Zhang B, Zhang Y, Li J, Su L, Zhu Z, Gu Q, Liu B and Yan M: CEACAM6 promotes gastric cancer invasion and metastasis by inducing epithelial-mesenchymal transition via PI3K/AKT signaling pathway. Plos One 9: e112908, 2014.

26. Ptak A, Hoffmann M, Gruca I and Barć J: Bisphenol A induce ovarian cancer cell migration via the MAPK and PI3K/Akt signalling pathways. Toxicol Lett 229: 357-365, 2014.

27. Zhang W, Glöckner SC, Guo M, Machida EO, Wang DH, Easwaran H, Van Neste L, Herman JG, Schuebel KE, Watkins DN, et al: Epigenetic inactivation of the canonical Wnt antagonist SRY-box containing gene 17 in colorectal cancer. Cancer Res 68: 2764-2772, 2008 .

28. Ying J, Poon F, Yu J, Geng H, Wong AH, Qiu GH, Goh HK, Rha SY, Tian L, Chan AT, et al: DLEC1 is a functional 3 p22.3 tumour suppressor silenced by promoter $\mathrm{CpG}$ methylation in colon and gastric cancers. Br J Cancer 100: 663-669, 2009.

29. Hanada N, Takahata T, Zhou Q, Ye X, Sun R, Itoh J, Ishiguro A, Kijima H, Mimura J, Itoh K, et al: Methylation of the KEAP1 gene promoter region in human colorectal cancer. BMC Cancer 12: 66, 2012

30. Migheli F and Migliore L: Epigenetics of colorectal cancer. Clin Genet 81: 312-318, 2012

31. Conacci-Sorrell M, Zhurinsky J and Ben-Ze'ev A: The cadherin-catenin adhesion system in signaling and cancer. J Clin Invest 109: 987-991, 2002

32. Parsons DW, Wang TL, Samuels Y, Bardelli A, Cummins JM, DeLong L, Silliman N, Ptak J, Szabo S, Willson JK, et al: Colorectal cancer: Mutations in a signalling pathway. Nature 436: 792, 2005

33. Burgering BM and Coffer PJ: Protein kinase B (c-Akt) in phosphatidylinositol-3-OH kinase signal transduction. Nature 376: 599-602, 1995

34. Ekstrand AI, Jönsson M, Lindblom A, Borg Å and Nilbert M: Frequent alterations of the PI3K/AKT/mTOR pathways in hereditary nonpolyposis colorectal cancer. Fam Cancer 9: 125-129, 2010.

35. Lau MT and Leung PC: The PI3K/Akt/mTOR signaling pathway mediates insulin-like growth factor 1-induced E-cadherin down-regulation and cell proliferation in ovarian cancer cells. Cancer Lett 326: 191-198, 2012.

36. Dilly AK, Ekambaram P, Guo Y, Cai Y, Tucker SC, Fridman R, Kandouz $M$ and Honn KV: Platelet-type 12-lipoxygenase induces MMP9 expression and cellular invasion via activation of PI3K/Akt/NF- $\mathrm{KB}$. Int J Cancer 133: 1784-1791, 2013.

This work is licensed under a Creative Commons Attribution-NonCommercial-NoDerivatives 4.0 International (CC BY-NC-ND 4.0) License. 